\title{
ENDOMORPHISMS OF A LEBESGUE SPACE
}

\author{
BY WILLIAM PARRY ${ }^{1}$ AND PETER WALTERS \\ Communicated by Calvin C. Moore, August 3, 1971
}

0 . Introduction. Considerable progress has been made in the classification of measure preserving transformations during the last thirteen years, reaching a high point with the recent work of Ornstein [1]. Most of this theory has concentrated on invertible transformations (automorphisms) since it was here that the essential problems awaited solution. Viewed as two-sided shifts on symbol spaces, an isomorphism between invertible transformations amounts to a faithful coding between their respective infinite messages. A new problem appears, however, if a coding which does not anticipate the future is required. From this point of view such a coding establishes a correspondence between their associated one-sided shifts. This is one motivation for pursuing the classification of transformations which are not necessarily invertible (endomorphisms).

No proofs will be given in this paper although it should be noted that one of the principal invariants mentioned here appears in [2]. We should also like to refer interested readers to the recent work of Versik [3], [4], to Rohlin's paper [5] and the closing remarks of Rohlin in [6]. (The question raised in the last paragraph of $[\mathbf{6}]$ has a negative answer.)

1. The index function. Throughout we shall be concerned only with Lebesgue spaces. A transformation $T$ of one space $\left(X_{1}, \mathscr{B}_{1}, m_{1}\right)$ to another $\left(X_{2}, \mathscr{B}_{2}, m_{2}\right)$ is called a homomorphism if $T X_{1}=X_{2}, T^{-1} \mathscr{B}_{2} \subset \mathscr{B}_{1}$ and $m_{1}\left(T^{-1} B_{2}\right)=m_{2}\left(B_{2}\right)$ for all $B_{2} \in \mathscr{B}_{2}$. If the two spaces coincide $T$ is called an endomorphism. If a homomorphism is invertible it is called an isomorphism and an endomorphism which is also an isomorphism is called an automorphism. As usual in measure theory, we do not distinguish between two objects which coincide a.e., and for this reason objects need only be defined a.e.

Using Rohlin's theory of measurable partitions it was established in [7] that if $T$ is a countable to one endomorphism of a Lebesgue space $(X, \mathscr{B}, m)$ then there is a null set $N$ with $T(X-N)=X-N$ and such that $T \mid X-N$ is positively measurable and positively nonsingular, i.e. $T \mid X-N$ maps measurable sets to measurable sets and null sets to null sets. We shall always assume that countable to one endomorphisms have been so modified.

AMS 1970 subject classifications. Primary 28A65.

Key words and phrases. Coding, Lebesgue space, exactness, Jacobian, discrete spectrum $\bmod \beta(T)$, coboundary.

${ }^{1}$ Research partially supported by NSF GP-15735. 
A trivial invariant suggests itself immediately viz. the number of inverse images of a point. Actually this is not quite an invariant. The appropriate function defined by a homomorphism $T: X_{1} \rightarrow X_{2}$ is the index $i_{T}\left(x_{2}\right)$ which equals the number of atoms in $T^{-1}\left(x_{2}\right)$ (with respect to the conditional measure $\left.m_{1}\left(\mid T^{-1} x_{2}\right)\right)$ if this measure is atomic and which equals $+\infty$ otherwise. (The effect of modifying a countable to one endomorphism is to remove points from $T^{-1}(x)$ with zero weight.)

If endomorphisms $T_{1}: X_{1} \rightarrow X_{2}, T_{2}: X_{2} \rightarrow X_{2}$ are isomorphic (i.e. there exists an isomorphism $F: X_{1} \rightarrow X_{2}$ with $\left.F T_{1}=T_{2} F\right)$ then $i_{T_{1}}\left(x_{1}\right)$ $=i_{T_{2}}\left(F x_{1}\right)$. We are interested in classifying endomorphisms whose index functions are isomorphic in the sense that $i_{T_{1}}\left(x_{1}\right)=i_{T_{2}}\left(F x_{1}\right)$ for some isomorphism $F: X_{1} \rightarrow X_{2}$.

2. Sequences of $\sigma$-algebras. Versik [3], [4] studies an endomorphism $T$ via its associated sequence of $\sigma$-algebras $\left\{T^{-n} \mathscr{B}\right\}$, and, more abstractly, studies decreasing sequences of $\sigma$-algebras $\mathscr{B}=\mathscr{B}^{0} \supset \mathscr{B}^{1} \supset \cdots$. Imitating the property of exactness $\left(\bigcap_{n} T^{-n} \mathscr{B}=\mathscr{N}\right.$ the trivial $\sigma$-algebra) he also imposes the condition $\bigcap_{n} \mathscr{B}^{n}=\mathscr{N}$.

If $\left(X_{i}, \mathscr{B}_{i}, m_{i}\right)(i=1,2)$ are Lebesgue spaces and $\left\{\mathscr{B}_{i}^{n}\right\}$ are corresponding decreasing sequences, they are said to be finitely isomorphic if for each $n$ there is an isomorphism $F_{n}: X_{1} \rightarrow X_{2}$ with $F_{n}\left(\mathscr{B}_{1}^{j}\right)=\mathscr{B}_{2}^{j}$, for $j=0,1, \ldots n$, and simply isomorphic if there is an isomorphism $F: X_{1} \rightarrow X_{2}$ such that $F\left(\mathscr{B}_{1}^{n}\right)=\mathscr{B}_{2}^{n}$ for $n \geqq 0$. If $T_{1}$ and $T_{2}$ are isomorphic then so are $\left\{T_{1}^{-n} \mathscr{B}_{1}\right\}$ and $\left\{T_{2}^{-n} \mathscr{B}_{2}\right\}$.

A decreasing sequence $\left\{\mathscr{B}^{n}\right\}$ with $\bigcap_{n} \mathscr{B}^{n}=\mathscr{N}$ is called dyadic if for each $n$ the conditional measures defined by $\mathscr{B}^{n+1}$ relative to $\mathscr{B}^{n}$ consist of two atoms of equal weight. An example of a dyadic sequence is provided by $\left\{T^{-n} \mathscr{B}\right\}$ where $T$ is the one-sided Bernoulli $\left(\frac{1}{2}, \frac{1}{2}\right)$ shift. Versik claimed in [3] that finitely isomorphic sequences (decreasing to $\mathscr{N}$ ) are isomorphic, but corrected this in [4] by producing two nonisomorphic dyadic sequences. A nondyadic example is easier to construct. In fact if $T_{1}$ is the one-sided Bernoulli $(p, q)$ shift $\left(p \neq \frac{1}{2}\right)$ and $T_{2}$ is the one-sided Markov shift with transition matrix $\left(\begin{array}{cc}p & q \\ q & p\end{array}\right)$ then the sequences $\left\{T_{1}^{-n} \mathscr{B}_{1}\right\}$ and $\left\{T_{2}^{-n} \mathscr{B}_{2}\right\}$ are finitely isomorphic but not isomorphic. (The nonisomorphism of $T_{1}$ and $T_{2}$ was first noted in $[\mathbf{8}]$.)

An alternative way of looking at the sequence $\left\{T^{-n} \mathscr{B}\right\}$ is as follows. Let $S, T$ be endomorphisms of $(X, \mathscr{B}, m)$. We say $S, T$ are sequentially equivalent if there are automorphisms $\phi_{1}, \phi_{2}, \ldots$ of $X$ with $\phi_{n+1} S=T \phi_{n}$, $n \geqq 1$.

Proposition 1. $S, T$ are sequentially equivalent if and only if $\left\{S^{-n} \mathscr{B}\right\}$, $\left\{T^{-n} \mathscr{B}\right\}$ are isomorphic. In fact $\phi_{1} S^{-n} \mathscr{B}=T^{-n} \mathscr{B}$ for $0 \leqq n \leqq N$ if and only if there exist automorphisms $\phi_{n}(1 \leqq n \leqq N+1)$ with $\phi_{n+1} S=T \phi_{n}$ $(1 \leqq n \leqq N)$. 
This proposition suggests studying the following sequence of groups associated with an endomorphism $T$. Let $H_{1}=\left\{\phi_{1}: \phi_{1}\right.$ is an automorphism such that $\phi_{2} T=T \phi_{1}$ for some automorphism $\left.\phi_{2}\right\}, G_{1}=\left\{\phi_{2}\right.$ : $\phi_{2} T=T \phi_{1}$ for some $\left.\phi_{1} \in H_{1}\right\}$, and if $H_{1} \supset H_{2} \supset \cdots \supset H_{n}$ and $G_{1} \supset G_{2} \supset \cdots \supset G_{n}$ are defined let $H_{n+1}=H_{1} \cap G_{n}$ and $G_{n+1}=\left\{\phi_{n+2}\right.$ : $\phi_{n+2} T=T \phi_{n+1}$ for some $\left.\phi_{n+1} \in H_{n+1}\right\}$. Let $H=\bigcup_{n} H_{n}, G=\bigcup_{n} G_{n}$. The map $\sigma: H \rightarrow G$ defined by $\sigma(\phi) T=T \phi$ is a homomorphism and $\sigma\left(H_{n}\right)=G_{n}, n \geqq 1$. The kernel of $\sigma$ is the group of all automorphisms which induce the identity on $T^{-1} \mathscr{B}$.

Proposition 2. Let $\phi$ be an automorphism. $\phi \in \bigcap_{n=0}^{N} \sigma^{-n}\left(H_{n+1}\right)$ if and only if $\phi T^{-n} \mathscr{B}=T^{-n} \mathscr{B}$ for $0 \leqq n \leqq N+1$. Therefore $\phi \in \bigcap_{n=0}^{\infty} \sigma^{-n}\left(H_{n+1}\right)$ if and only if $\phi T^{-n} \mathscr{B}=T^{-n} \mathscr{B}$ for $n \geqq 0$.

Suppose now that $T$ is the one-sided Bernoulli $\left(\frac{1}{2}, \frac{1}{2}\right)$ shift. In this case all the groups $H_{n}(n \geqq 1)$ coincide and consist of all automorphisms of the form $\phi\left(x_{0}, x_{1}, \ldots\right) \equiv \phi(x) \equiv \phi\left(x_{0}, T x\right)=\left(x_{0}+\rho(T x), \psi(T x)\right)$, where $x_{i}=0$ or 1 and addition is to be interpreted $\bmod 2, \rho: X \rightarrow\{0,1\}$ is any measurable map and $\psi: X \rightarrow X$ is any automorphism. The groups $G_{n}$ coincide and consist of all automorphisms. The group of all automorphisms $\phi$ with $\phi T^{-n} \mathscr{B}=T^{-n} \mathscr{B}(n \geqq 0)$ consist of all automorphisms of the form

$$
\phi(x)=\left(x_{0}+\rho_{1}(T x), x_{1}+\rho_{2}\left(T^{2} x\right), x_{2}+\rho_{3}\left(T^{3} x\right), \ldots\right)
$$

where each $\rho_{i}: X \rightarrow\{0,1\}$ is measurable. (Every map of this form is measure preserving but not necessarily one-one.) From this, one can deduce that the group of all automorphisms commuting with $T$ consists of all automorphisms of $X$ of the form $\left(x_{0}, x_{1}, \ldots\right) \rightarrow\left(x_{0}+\rho(T x)\right.$, $x_{1}+\rho\left(T^{2} x\right), \ldots$ ) where $\rho: X \rightarrow\{0,1\}$ is measurable.

On the other hand when $T$ is the one-sided Bernoulli $(p, q)$ shift $\left(p \neq \frac{1}{2}\right)$ then all the groups $H_{n}, G_{n}$ are trivial.

It is easy to find examples of exact endomorphisms $S, T$ with isomorphic sequences $\left\{S^{-n} \mathscr{B}\right\},\left\{T^{-n} \mathscr{B}\right\}$ and differing entropies. If $K^{2}=K \times K$ denotes the 2-torus with multiplicative notation, let $T(z, w)=\left(z^{2}, w^{2}\right)$ and $\phi(z, w)=\left(z^{2} w, z w\right)$ ( $\phi$ is an automorphism). Put $S=\phi T=T \phi$. Then $S^{-n} \mathscr{B} \equiv T^{-n} \mathscr{B}$ and $S, T$ have different entropies. Later (see $\S 4$ ) we give two nonisomorphic endomorphisms $S, T$ with isomorphic sequences and equal entropy. (In fact we can choose them with $S^{2}=T^{2}$.)

3. The Jacobian. Closely related to the sequence $\left\{T^{-n} \mathscr{B}\right\}$ and, in some sense, dual to the index there is the Jacobian $j_{T}$ of an endomorphism or, more generally, of a homomorphism [2]. (The index counts the number of points in the inverse images, whereas the Jacobian measures how a point breaks up into different points under the inverse.) $j_{T}(x)$ $=1 / m\left(x \mid T^{-1} T(x)\right)$ where $m\left(\mid T^{-1} y\right)$ is the conditional measure on the set 
$T^{-1} y$. If $T$ is countable to one (i.e. $i_{T}$ is finite or countably infinite), there exists a countable partition $\alpha=\left(A_{1}, A_{2}, \ldots\right)$ such that $T \mid A_{i}$ is one-one. Then $d m\left(T \mid A_{i}\right) / d m$ is well defined for $i=1,2, \ldots$ and $j_{T}=$ $\sum_{i=1}^{\infty} \chi_{A_{i}} d m\left(T / A_{i}\right) / d m$. Note that $T$ is an automorphism if and only if $j_{T}=1$ a.e.

If $S: X_{1} \rightarrow X_{2}, \quad T: X_{2} \rightarrow X_{3}$ are homomorphisms then $j_{\text {ToS }}(x)$ $=j_{T}(S x) j_{S}(x)$. In particular if $S$ is an isomorphism, $j_{T \circ S}(x)=j_{T}(S x)$, and if $T$ is an isomorphism $j_{T} \circ S(x)=j_{S}(x)$. If $S, T$ are endomorphisms and $\phi, \psi$ are isomorphisms with $\phi S=T \psi$ then $j_{S}=j_{T} \psi$. If $S, T$ are endomorphisms of $X_{1}, X_{2}$ respectively and if $\phi$ is a homomorphism with $\phi S=T \phi$ and $j_{S}=j_{T} \phi$ (i.e. $S$ is one-one on fibres) then $j_{\phi}(S)=j_{\phi}$. In this case, if $S$ is ergodic $j_{\phi}$ is constant so that $\phi$ is exactly $k$ to 1 and $j_{\phi}=1 / k$, or $\phi$ is not countable to one.

Let $\beta(T)$ be the smallest $\sigma$-algebra such that $T^{-1} \beta(T) \subset \beta(T)$ with respect to which $j_{T}$ is measurable. If $S$ is isomorphic to $T(\phi S=T \phi)$ then $\beta(T)=\phi \beta(S)$. One way to show that the one-sided Markov shift with transition matrix $\left(\begin{array}{cc}p & q \\ q & p\end{array}\right)\left(p \neq \frac{1}{2}\right)$ is not isomorphic to the one-sided Bernoulli $(p, q)$ shift is to show that the corresponding $\beta(T)$ are not isomorphic. $\beta(T) \neq \mathscr{B}$ for the former and $\beta(T)=\mathscr{B}$ for the latter. (If $p=q=\frac{1}{2}$ then $\beta(T)=\mathscr{N}$.)

Proposition 3. If $\beta(T)=\mathscr{B}$ the identity is the only automorphism commuting with $T$.

4. Discrete spectrum $\bmod \beta(T)$. An endomorphism $T: X \rightarrow X$ is said to have discrete spectrum $\bmod \beta(T)$ if $\mathscr{G}=\left\{f:|f|=1\right.$ and $f T \mid f \in L^{2}(\beta(T))$, $f$ measurable $\}$ spans $L^{2}(\mathscr{B}) . \mathscr{G}$ forms a group under multiplication and its members are called eigenfunctions $\bmod \beta(T) . \mathscr{H}=\{f T / f: f \in \mathscr{G}\}$ is called the group of eigenvalues $\bmod \beta(T)$.

Let $S: Y \rightarrow Y$ denote the factor endomorphism of $T$ corresponding to $\beta(T)$, where $T$ is ergodic and has discrete spectrum $\bmod \beta(T)$. Then $X$ is isomorphic to $Y \times G$ where $G$ is the dual of the countable group $\mathscr{G} /\{f:|\not|$ $\left.=1, f \in L^{2}(\beta(T))\right\}$. Moreover, $T$ has an isomorphic representation as $T(y, g)=(S y, \phi(y)+g)$ where $\phi: Y \rightarrow G$ is measurable. Let $T_{\psi}$ denote the endomorphism $T_{\psi}(y, g)=(S y, \phi(y)+\psi(y)+g)$. Using Proposition 3 one can prove:

Proposition 4. If $T$ is ergodic with discrete spectrum $\bmod \beta(T)$, then $T_{\psi}$ is isomorphic to $T$ only if $\psi$ is a coboundary of $T$ (i.e. $\psi=h(T)-h$ for some measurable $h: X \rightarrow G)$. The only automorphisms commuting with $T$ have the form $(y, g) \rightarrow(y, c+g), c \in G$.

COROLlaRY 1. $T_{g_{0}}$ is isomorphic to $T$ only if $\gamma\left(g_{0}\right)$ is an eigenvalue of $T$ for each $\gamma \in \hat{G}$, the character group of $G\left(g_{0} \in G\right)$. 
If $S$ is an exact endomorphism $\left(\bigcap_{n} T^{-n} \beta(T)=\mathscr{N}\right)$ and $T$ is weakmixing (which is "usually" the case [9]) then $T$ is exact. By the above corollary if $T$ is weak-mixing then the endomorphisms $T_{g_{0}}$, for varying $g_{0}$, are mutually nonisomorphic. They have identical sequences $\left\{T_{\mathbf{g}_{0}}^{-n} \mathscr{B}\right\}$ and identical entropy.

By choosing a weak-mixing circle extension of (for example) the Bernoulli $(p, q)$ shift $\left(p \neq \frac{1}{2}\right)$ and choosing $g_{0}=-1$ we get:

COROLlary 2. There are nonisomorphic exact endomorphisms $S, T$ with $S^{2}=T^{2}, S^{-n} \mathscr{B}=T^{-n} \mathscr{B}$ for all $n \geqq 0, j_{S} \equiv j_{T}$ and $\beta(S)=\beta(T)$.

\section{REFERENCES}

1. D. S. Ornstein, Bernoulli shifts with the same entropy are isomorphic, Advances in Math. 4 (1970), 337-352. MR 41 \# 1973.

2. W. Parry, Entropy and generators in ergodic theory, Benjamin, New York, 1969. MR 41 \# 7071.

3. A. M. Versik, A theorem on the lacunary isomorphisms of monotone sequences of partitions, Funkcional. Anal. i Priložen. 2 (1968), no. 3, 17-21 = Functional Anal. Appl. 2 (1968), no. 3, 200-203.

4. , Decreasing sequences of measurable partitions and their applications, Dokl. Akad. Nauk SSSR 193 (1970), 748-751 = Soviet Math. Dokl. 11 (1970), 1007-1011.

5. V. A. Rohlin, Exact endomorphisms of a Lebesgue space, Izv. Akad. Nauk SSSR Ser. Mat. 25 (1961), 499-530; English transl., Amer. Math. Soc. Transl. (2) 39 (1964), 1-36. MR 26 \# 1423.

6. Uspehi Mat. Nauk 22 (1967), no. 5 (137), 3-56 = Russian Math. Surveys 22 (1967), no. 5, 1-52. MR 36 \# 349.

7. P. Walters, Roots of $n: 1$ measure-preserving transformations, J. London Math. Soc. 44 (1969), 7-14. MR 38 \# 1236.

8. H. Furstenberg, Disjointness in ergodic theory, minimal sets, and a problem in Diophantine approximation, Math. Systems Theory 1 (1967), 1-49. MR 35 \# 4369.

9. R. Jones and W. Parry, Compact abelian group extensions of dynamical systems, Compositio Math. (to appear).

Department of Mathematics, University of California, Berkeley, California 94720 Department of Mathematics, University of Maryland, College Park, Maryland 20742

Department of Mathematics, University of Warwick, Coventry, England 